\title{
Understanding organizational context and heart failure management in long term care homes in Ontario, Canada
}

\author{
Jill Marcella ${ }^{1}$, Jayanthini Nadarajah ${ }^{2}$, Mary Lou Kelley ${ }^{3 *}$, George A. Heckman ${ }^{4}$, Teresa D'Elia ${ }^{4}$ \\ Sharon Kaasalainen ${ }^{5}$, Patricia H. Strachan ${ }^{5}$, Robert S. McKelvie ${ }^{6}$, lan Newhouse ${ }^{3}$, \\ Paul Stolee ${ }^{4}$, Carrie A. McAiney ${ }^{7}$, Catherine Demers ${ }^{6}$

\footnotetext{
${ }^{1}$ School of Social Work, Centre for Education and Research on Aging and Health, Lakehead University, Thunder Bay, Canada

${ }^{2}$ School of Medicine, University of Ottawa, Ottawa, Canada

${ }^{3}$ School of Social Work and Northern Ontario School of Medicine, Centre for Education and Research on Aging and Health, Lakehead University, Thunder Bay, Canada; ${ }^{*}$ Corresponding Author: mlkelley@lakeheadu.ca

${ }^{4}$ School of Public Health and Health Systems, Faculty of Applied Health Sciences, University of Waterloo, Waterloo, Canada

${ }^{5}$ School of Nursing, McMaster University, Hamilton, Canada

${ }^{6}$ Population Health Research Institute, McMaster University, Hamilton, Canada
} \\ ${ }^{7}$ Department of Psychiatry and Behavioural Neurosciences, Hamilton, Canada
}

Received 1 August 2012; revised 31 August 2012; accepted 10 September 2012

\section{ABSTRACT}

Objective: To assess current heart failure (HF) care processes and organizational context in long-term care (LTC) homes as a prelude to adapting the Canadian Cardiovascular Society (CCS) HF guidelines for use in these settings. Methods: This research reports on the results of thirteen focus groups ( $N=83$ participants; average of 60 minutes duration) conducted in three Ontario LTC homes to better understand how HF was managed and how organizational context impacted care. Participants included physicians, nurse practitioners, registered nurses, registered practical nurses, and personal support workers. Results: Focus group findings revealed that the complexity of the LTC environment presents challenges for managing HF. Most residents have multiple advanced chronic conditions that must be managed simultaneously. Culturally, LTC is first and foremost a resident's home where residents may choose not to comply with care recommendations. Staff routines, scopes of practice, professional hierarchies, available resources and government regulations limit flexibility in providing care. Staff lacked knowledge, skills and resources for managing HF. Nevertheless, all staff viewed LTC as the preferred place for managing HF, avoiding residents' hospitalizations wherever possible. These data suggest that strategies for improving LTC staff communication and education, strength- ening existing relationships between staff, family, residents and community resources, and acquiring additional resources in LTC homes have the potential to improve HF management in this setting. Conclusion: LTC is a complex and dynamic environment that presents many challenges for providing care for residents. This research provides the foundation for subsequent work to develop and test implementation strategies to manage HF in LTC, which are consistent with the CCS HF guidelines and are feasible within LTC staff's work routines, capacities and resources.

Keywords: Heart Failure; Long-Term Care; Organizational Context; Focus Groups; Interprofessional; Disease Management

\section{BACKGROUND}

Increased life expectancy and health care system pressures have resulted in many frail seniors with complex health challenges being admitted to long-term care (LTC) homes [1]. Heart failure (HF) is a chronic, progressive illness that affects at least $20 \%$ of LTC residents [2]. Seniors with HF are more likely to be frail and suffer from cognitive and functional impairment, characteristics that complicate the management of HF and that are associated with worse health outcomes [3]. HF may account for up to $20 \%$ of transfers of LTC residents to hospital [4-6]. The impact of hospitalization on frail LTC residents can be significant, with prolonged stays in emer- 
gency departments and hospital wards, exposure to the risks of delirium, functional decline and other hospitalacquired complications, and potentially long delays in transfer back to their home or LTC home [6,7]. Many of these admissions and their associated complications might be prevented through more optimal management of $\mathrm{HF}$ in LTC [8-12].

Specific protocols and clinical guidelines are used infrequently in LTC [13]. A small number of non-randomized studies evaluating HF management interventions in LTC suggest that such initiatives can reduce health service utilization [14-19]. However, these interventions relied on access to substantial resources beyond those normally available in LTC homes and targeted less frail residents expected to return to the community. Importantly, none of these protocols were designed specifically to reflect the unique organizational context of the LTC setting. Concerns that have been identified in LTC focus on staffing, communication, resources and the changing scope of practice of health care professionals $[1,20]$. All of these concerns emphasize the need for HF management protocols that are specifically adapted for LTC.

This paper reports on the results of phase two of a program of research that aims to develop care processes for HF in LTC that are consistent with the Canadian Cardiovascular Society (CCS) HF guidelines [21], that respect the capabilities and existing work routines of LTC staff, and that take into consideration the characteristics of the LTC environment. Data from phase one, LTC guideline development, have been published previously [20]. Phase two consisted of focus groups with long term care home staff to examine current care practices, barriers and facilitators to HF management in LTC, and to make recommendations on how to improve $\mathrm{HF}$ care in LTC.

\section{METHODS}

\subsection{Data Collection}

The focus group phase (phase two) of this study utilized a qualitative descriptive design [22-24]. Focus group interviews were conducted on site in three LTC homes in Ontario, Canada. These sites were purposefully selected to offer variability with respect to setting (southern vs. northern Ontario), size (251, 125 and 96 beds, respectively), affiliation (university-affiliated vs. non-university affiliated) and ownership (public vs. private ownership, and profit vs. not-for-profit). These characteristics of LTC homes have been shown to be relevant to HF management and outcomes [25-29]. Each site employs 22 to 25 licensed nurses, including Registered Nurses (RN) and Registered Practical Nurses (RPN), and over 30 personal support workers (PSW). Family physi- cians (MD) and Nurse Practitioners (NP) provide coverage for each site and other surrounding LTC homes. None of these sites had in place a dedicated HF management program.

Thirteen focus groups were conducted that included eighty three participants ( $\mathrm{n}=29 \mathrm{RNs}$ and RPNs, $\mathrm{n}=33$ PSWs, $n=14$ MDs, $n=7$ NPs). The target sample size for each focus group was 6 to 10 [22-24]. Purposive sampling, with the help of care directors from each facility to identify and recruit participants, was used to identify MDs, NPs, pharmacists, RNs, RPNs and PSWs who had substantial experience working in long term care homes and were effective communicators. The sample represented the range of disciplines who participate in the care of residents with heart failure in long term care homes. Focus groups were organized according to discipline in order to maximize participant compatibility and reduce the risk that real or perceived power imbalances might curtail the sharing of ideas. As individual homes were served by a small number of physicians, MDs serving other local homes, as well as NPs and pharmacists, were recruited to achieve the desired sample size. A trained moderator facilitated discussions, and a second person observed nonverbal communication within groups and took additional field notes [22]. A semi-structured interview guide specific to each staff role was developed to explore staff perceptions of 1) current practices with respect to HF management; 2) potential barriers to the implementation of HF care processes in LTC; 3) facilitators for the implementation of HF care processes in LTC; and 4) supports and processes required to successfully implement HF care processes in LTC.

\subsection{Analysis}

Focus group interviews were digitally recorded, transcribed verbatim and verified by research assistants through comparison of written transcripts with the audio recordings. An inductive approach was used in analyzing focus group data and a three-level analysis was performed independently by two authors/research assistants who had different perspectives, one a physician (JN) and one a social worker (JM) who had worked many years in LTC homes [30]. These research assistants coded the first focus group simultaneously to compare the results of their analysis. Any coding differences were discussed between themselves and with a senior researcher (MK) to create consensus and finalize the coding structure. The remainder of the focus groups were then coded by one of the research assistants and reviewed by the second research assistant and the senior researcher to ensure reliability and validity.

The first level of analysis consisted of line by line inductive coding of the transcript to identify all expressed 
ideas. The second level of analysis grouped the ideas into higher-order themes, and the third level of analysis the themes were grouped into categories. For each category, the corresponding themes and subthemes were linked and organized using a concept map to display the relationships amongst ideas within the data; focus group quotations were extracted to provide evidence to support the themes. As themes were similar across participant groups, these were combined in the reporting of the analysis. Data saturation, whereby successive focus group data confirmed ideas identified by previous interviews $[31,32]$, was considered to have been reached with the current sample size.

To ensure rigour, the preliminary results were subjected to peer-review by the 12 member research team in the form of a report to discuss interpretation and conclusions. Focus group results were then presented to the LTC staff in a workshop, and this member checking validated the conclusions of the analysis. Peer review and member checking were employed as the rigour of an inquiry such as this is judged, in part, by the story's credibility and applicability. The credibility of these research results rests on others seeing and accepting the relationship between the facts and the reasoning [33]. The applicability is determined when the findings resonate with the experience of the staff who understand and work in long term care [33] as they did during the workshop described.

Ethics clearance was provided by the research ethics boards of McMaster University and Lakehead University.

\section{RESULTS}

The results of the focus group data analysis illustrate the complexity of the LTC environment. Findings are reported using three categories related to the context of heart failure management in long term care homes: current care practices, barriers and facilitators to managing health failure and LTC staff perspectives about strategies to improve HF management. Within each of these categories, multiple themes are identified with supporting evidence taken from the participant data.

\subsection{Current Care Practices}

Within this category, four themes were identified that influenced current practice: the quality of preliminary clinical assessments; care protocols for other conditions; communication patterns; and changing policy requirements from the Ontario Ministry of Health and Long Term Care (MOHLTC). Each of these is elaborated in the following sections.

\subsubsection{Preliminary Clinical Assessment}

Participants stated that the quality of the preliminary assessment of HF depends on front line staff (PSWs) recognizing changes in the resident's normal functioning and then reporting these changes to a member of the registered staff, namely, a RN or RPN. Reports of such changes alert nurses that a problem exists and could trigger a call to the physician. Thus, the ability of the PSW to identify relevant changes and the consistency of their reporting are key factors in the quality of the preliminary assessment. This valuable role of the PSW is expressed by one physician, who stated:

"...What's more important is change and the people that know it best are the people that are with the patient on a daily basis, and it's not the Doctor. It's not the RN. It's the basic Health Care Aide [PSW] that knows that patient and knows when something is changing...” (MD)

Most residents entering LTC have multiple health problems that complicate the assessment and management of HF. The key role of PSWs as front-line staff was identified above. However, when asked to describe the signs and symptoms of HF in the focus groups, our data indicated that most PSWs have insufficient knowledge of the signs and symptoms on which to base appropriate interventions. Many PSWs could not easily describe or were mistaken about the symptoms of HF and how to respond. For example, one PSW described the signs of $\mathrm{HF}$ as

"A lot of times it seems there's dehydration, and the first thing you do with them is make sure they're drinking. Bowels are a big part of it." (PSW)

By contrast, in their focus groups, nurses and physicians suggested that the knowledge needed by PSWs in order to alert them reliably about important changes in HF patients should include knowledge about basic HF physiology and clinical manifestations, the resident's past medical history, and the ability to accurately convey this information to a nurse or physician. This degree of knowledge greatly exceeds that of PSWs who participated in our study.

\subsubsection{Care Protocols}

Participants indicated that there are a number of currently established generic protocols that impact on, and may be a barrier to, implementing new ones to manage HF. Meal provision in LTC, which often includes prepackaged items, soups and gravies with high sodium content, was considered a barrier to HF care. Physicians and PSWs spoke about the difficulty in deviating from standardized menus in the LTC home and emphasized that residents have the right to add salt at the table and not comply with heart healthy diets. In this respect, LTC implements the philosophy that it is a resident's home and not a health care institution. One PSW stated:

"You know gravy is not one of the best things to eat every day...they're [served gravy] often at two meals a 
day. So lunch and supper, gravy with mashed potatoes. And if you're on a ground or pureed diet, you're getting it every time." (PSW)

No standard HF management protocols were in place in any of the participating LTC homes. For example, an emergency medication box that included injectable furosemide, benzodiazepines and narcotics was available in two of the homes but not in the third. Criteria and processes to decide when to transfer a resident to hospital varied across homes. In general, with respect to HF management, the scope of practice of each care discipline determines what tasks staff can perform and how changes in residents are to be monitored. For example, some PSWs are given the responsibility to monitor residents' responses to medication although their job description does not include assessment or care planning, which are within the scope of practice of nurses:

"Sometimes we are made aware that they [residents] are put on a fluid pill or what have you and we have to watch how many times and how often they go to the bathroom and that sort of thing." (PSW)

\subsubsection{Communication Patterns within LTC Homes}

The importance of communication among all care providers was identified by participants as integral to successful HF management. Communication patterns in LTC were described as hierarchical in nature with the physician at the top of the communication chain and the PSW at the bottom. PSWs rarely communicate directly with physicians despite some physicians acknowledging the value of information received from PSWs. The professional hierarchy was described as follows:

"You have top down, there is always a physician that is in charge of their care that comes once a week. Also somebody is on call all the time if necessary, you have the RN, and you have the RPN and then you have us." (PSW)

Communication between shifts was identified as key in transmitting pertinent information. Participants identified that communication was accomplished through a variety of ad hoc, locally-devised solutions, such as verbal reports, flow sheets and communication logs. Several participants raised concerns about the quality and completeness of information being communicated, such as these comments:

"That's a communication thing again, right. That's a hard one when a new resident, we don't know the care or how to go about things even if they've been on another floor and you come to a different floor, like the communication for care is just like nothing, sometimes." (PSW)

"You're trying to do the best job you have with the information you have and I mean we kind of put bits and pieces together for care..." (PSW)
Some participants identified reliance on informal communication as equally beneficial as formal pathways of shift change reporting, though others felt that such methods were exclusionary of some staff roles and thus inefficient. One PSW noted:

"The two RPNs do a verbal at exchange of shift. So a lot of times that information isn't shared with other staff. So then there's that due diligence part on us looking at the information on our own and finding out what's going on." (PSW)

\subsubsection{Changing Policy Requirements from MOHLTC}

Long term care homes are highly regulated regarding resident care and these regulations were perceived as often changing. Changing policies from the MOHLTC were viewed by participants as confusing since they were often unaware of details in policy changes or what new policies were in effect. Several participants commented on the disruption of established care practices caused by the implementation across Ontario of new MOHLTC policies and regulations regarding general care provision in LTC. Participants felt overwhelmed with new expectations placed upon them from the MOHLTC, some of which were felt to distract from the provision of clinical care. One RPN offered the following example:

"The thing is that since this [new report system] was implemented the difference with the report is we've had a super increase in paper work. They've [management] taken away our first flow sheets because the government has instituted a much longer RAI [the MDS 2.0 Resident Assessment Instrument] system. So it's just putting in your lap all these things to do and less time to be with your residents." $(R P N)$

Examples were provided about certain policies being contrary to the best interests of residents with HF. One PSW recalls expressing concerns to a nurse about the ability of one particularly dyspneic resident with a history of HF to attend a meal in the facility dining-room. The PSW was reminded that all meals are to be taken in the dining room. However, the PSW took it upon herself to return the resident back to her room as she was too ill to remain in the dining room; the resident died later that day.

Participants said that external government policy and regulations influence current care practices in LTC. Staff described feeling overwhelmed by care expectations and confused by existing regulations. It was noted that any efforts to introduce new care protocols and expectations would need to be carefully integrated into the existing regulations and avoid adding to the staff sense of being overwhelmed.

The care of residents in LTC homes occurs in an environment that is characterized by the current practices and 
processes that have been discussed in this section. These existing care protocols affect current care practices for HF management in LTC. In addition to the general environmental characteristics identified in LTC, participants in our study identified specific barriers to managing HF. The various types of LTC staff, each with different scopes of practice, make implementing assessment and care protocols a challenge. These data emphasize the importance of optimal interprofessional communication in the assessment and management of HF in LTC, challenges to which are described below. The issue of communication influences current care practices in HF. In LTC, information needs to be shared by many staff and across shifts, however, staff expressed that communicating clear and consistent messages was difficult. Communication patterns and issues impact the assessment and treatment of residents' medical needs, including HF.

\subsection{Barriers and Facilitators to Managing HF in LTC}

Participants spoke freely of the challenges faced by staff in the participating LTC homes and how these challenges impact the provision of care. Four themes were identified as organizational barriers to providing $\mathrm{HF}$ management in LTC: staffing and scope of work; insufficient communication with hospitals; lack of resources; and the tension between respecting resident autonomy and implementing medical best practices. Each of these is elaborated upon in the following section.

Participants also identified a number of resources that facilitate the management of HF in LTC. The use of existing LTC procedures such as communication books, resident care conferences, checklists and the RAI MDS 2.0 (Resident Assessment Instrument Minimum Data Set) were identified by participants as potentially aiding in the transmission of information, monitoring, observing and treating HF symptoms. An interprofessional team approach with facilitated access to the physician was viewed as promoting continuity and consistency in care.

\subsubsection{Staffing and Scope of Practice}

All participants commented on the changing scopes of practice and job descriptions of LTC staff. There are fewer RNs working in LTC and their role includes more administrative tasks, whereas clinical skills which are needed in HF management are not utilized to their full capacity. One RN summarized this frustration:

"...We are not using the skills that we were trained to use by sitting at the computer typing a bunch of paperwork that nobody reads. ...we're the people that are responsible to make sure that if a resident needs a proper assessment it gets done and acted upon." (RN)

The RPN practice has grown to include medication administration, expanded clinical assessments and dressing changes, tasks previously assumed by RNs. All participants acknowledged that PSWs know residents best, but that the duties they are required to perform focuses primarily on the provision of activities of daily living. Participants commented that the information that could be shared by PSWs with registered staff was not always incorporated effectively, if at all, in care planning and decision-making:

"Actually sometimes it is the PSWs who are picking it [changes] up because they know they're the primary contact and they know any change and they'll tell you." (MD)

"We're relying on information coming to us. So if the PSWs aren't identifying factors, if they can't identify it to the RPNs or the RPNs can't identify issues, if we're not told information, we can't deal with it." $(R N)$

Participants acknowledged that the clinical profile of residents entering LTC has changed and that the acuity and complexity of health problems, characterized by increased frailty and multiple comorbid conditions, has increased over the years. The time required to organize the care of such residents, including assessment, and development and implementation of care plans, was perceived as often exceeding the scope of practice of LTC staff, and thus presents a barrier to managing complex conditions such as HF. As one physician noted:

"In general patients are more...complicated. Even in a place like this one...they're on a myriad of medications which may change. It's complicated...they're [LTC homes] very akin to hospitals, acute hospitals.” (MD)

Participants indicated feeling disadvantaged by a lack of clinical experience in managing multiple chronic health concerns. Participants perceived $\mathrm{HF}$ as a condition that is generally diagnosed and managed in acute care. LTC staff felt particularly disadvantaged by having little or no experience managing HF, either in acute care or in LTC.

\subsubsection{Insufficient Communication with Hospitals}

Participants identified significant communication problems between LTC homes and outside agencies. For example, participants identified a lack of information returning with the residents from hospital, with little or no rationale provided for medication changes, or information provided on how to follow-up and address abnormal test results. Participants perceived that such poor communication was particularly problematic with respect to the ongoing management of HF in complex LTC residents. One participant spoke of the importance of this communication when she stated that:

“...LTC staff spends a good amount of time filling out that beautifully done transfer record. They send all that pertinent information to the hospital, it comes back, theirs is the left side, the hospital is to fill out the right side, it 
comes back blank. I would say 99\% of the time it comes back blank. The specialist at the hospital might well dictate a note and will copy the family physician who may get it three, three and a half, four weeks later to his office. So you're working with no information...” (NP)

\subsubsection{Lack of Resources}

Participants identified a lack of available resources in LTC such as diagnostic equipment, access to timely diagnostic testing, and challenges with staff continuity and staffing shortage. Timely access to diagnostic testing presents a significant challenge in LTC, where many of the diagnostic tests required to identify and manage $\mathrm{HF}$ are not on-site (such as radiology) or readily available. This means that:

"...If something happens on a day that's not a lab day...we either have to send the person out or make special arrangements with the director of care to have the lab come in and do a stat call. Sometimes they can, sometimes they can't." (RPN)

Many participants spoke of the human resource challenge in LTC. It was indicated that staffing shortages often occur particularly on weekends and lead to further lack of care continuity when staff are moved to different units to accommodate the shortage. The shortage presents a challenge for RNs who spend time looking for replacement staff, leaving them less available to provide clinical support. One physician explained:

"I go in on weekends...and there's hours spent every Saturday and Sunday morning looking for staff because nine times out of ten, staff are phoning that they can't come in because they are sick...So every weekend, they're always short staffed. The nurse on call spends two or three hours phoning and leaving messages, trying to get staff." (MD)

Participants perceived that appropriate and comprehensive geriatric care was a resource that was missing in acute care settings. The acute care environment was deemed unable to appropriately accommodate the complex needs of the frail LTC resident, leading to misdiagnosis and the prescribing of inappropriate treatments. For example:

"Everyone going into emerg [emergency department] gets urine done first. So when the result comes back, off with the rest of the thinking. I've had someone sent because they fell and broke their shoulder and they were sent back with a UTI [diagnosis of a urinary tract infection]...I think it's the first positive result that reaches the emergency then they stop thinking." (MD)

\subsubsection{The Tension between Respecting Resident Autonomy and Implementing Medical Best Practices}

Focus group participants described that reconciling the principles of resident autonomy with best practices for HF could serve as both a barrier and a facilitator to HF management in LTC. All accepted that residents or designated substitute decision-makers have the inalienable right to decide whether or not to adhere with recommendations from the health team regarding medications, diet, or the decision to transfer to hospital. However, families often expressed priorities or expectations that the care team felt were at odds with the best interests of the resident. Examples ranged from the provision of sodiumladen snacks perceived by families as "treats", even though edema and dyspnea might be worsened, to last minute decisions by families to request a transfer to acute care, despite previous advance directives to the contrary. This tension was expressed in the following quotations:

"He [the resident] could make his own decisions and if he didn't want them [diuretics for heart failure] he wouldn't take it. And so he was managed well from our point of view [respect of resident autonomy], but his condition wasn't managed well because he was refusing [his diuretics].” (NP)

"There's advance directives that's very important, there's also the family too and sometimes expectations of the family can be what drives your decision and there's sometimes a conflict with what you feel what the patient wants and what the family wants but you're the one that's going to end up dealing with the family." (MD)

Most participants emphasized the importance of engaging residents and families in conversations about care and educating them about best practices for management of $\mathrm{HF}$ and other medical conditions common in LTC homes. Staff also stated that families can be a great support to the health care team when they are fully engaged in the care plan.

\subsection{LTC Staff Perspectives on Improving HF Management}

Focus group participants were asked to offer perspectives on how to improve HF management in LTC. The analysis of these data identified several theme areas, specifically with respect to communication strategies, building better interprofessional relationships, addressing educational needs of LTC staff, and providing additional resources. These data offer guidance for successful implementation of new care protocols from the perspective of the staff themselves. Importantly, these data identified a strong desire among all LTC staff to improve the management of HF among LTC residents, and specifically to reduce the need to access acute care services.

Participants indicated that keeping residents in LTC to receive treatment for $\mathrm{HF}$ was often preferred to sending residents to hospital. Participants considered themselves 
more sensitive than acute care staff to the needs and concerns of residents, who themselves have expressed a preference for familiar LTC staff and environment. The acute care setting is considered to be confusing and less responsive to the needs of the resident. The perceived dangers of transferring residents to hospital were expressed by a physician:

"When a patient is admitted to the hospital after a few days, they've gotten worse. If they stay in emerg [the emergency department], the noise, the delirium sets in, they get confused, they get bed sores, they fall, they break hips, and they get more de-conditioned." (MD)

\subsubsection{Improving Communication Strategies}

Participants recognized that successful communication among staff created opportunities for more optimal assessment and management of residents. Staff recognized that HF management was clearly improved when changes in a resident's condition were communicated promptly and received a timely response from the physician or nurse, or when information from the hospital or community resource was readily available. For example:

"Liaising with heart failure nurses is a recipe for good management because they have such an intricate knowledge of the medications. They know how to communicate that knowledge to the family and resident if they are able to." (NP)

Strategies to promote and improve effective communication among LTC staff and with residents and families were identified as a critical area of focus in moving forward with the implementation of care processes for HF, or for that matter any other health condition, in LTC. Participants believed that the organizational structure of LTC creates unique challenges to effective communication between staff; these stem from an entrenched hierarchical power structure, shifting roles among RNs, RPNs and PSWs, and reliance on ad hoc, untested, and disjointed communication methods particularly around shift change. In addition, a greater interprofessional emphasis during physician visits was identified as a potential and powerful approach to improving communications among LTC staff and ultimately improving resident outcomes. Improving communication with residents and staff, for example with respect to educating residents about HF management modalities, was seen as another potential mechanism for improving resident adherence to HF therapies.

Participants perceived that improving communication between LTC staff and external agencies, such as acute care or HF clinics, could improve resident care and outcomes. Improved communication strategies that include all staff, including PSWs, were universally identified by all focus group participants as a priority in the development of care processes for HF.

\subsubsection{Building Better Interprofessional Relationships}

Participants acknowledged how interprofessional collaboration is being promoted in LTC through a variety of MOHLTC initiatives and this is viewed as a facilitator to better care. Opportunities to apply interprofessional teamwork include physician rounds, as well annual family conferences, in which the resident care plans and advance directives are discussed. Participants identified that regular contact with physicians and a good working relationship between interprofessional members of the team facilitated HF management:

"...There was a team approach. We had to do inservice with RPNs, the registered staff as well as the PSWs, and talk about food balance and expectations with palliation and congestive heart failure. What our expectations were and what we expected to have on an outcome." (NP)

All focus group participants endorsed the need to strengthen existing relationships between different LTC staff roles, and between LTC staff and residents, families, as well as with other community programs and resources, such as nurse practitioners, acute care hospitals, and LTC placement agencies. The complexity of health care needs of residents with HF requires strong multilateral, supportive relationships among all individuals involved in the care of LTC residents with HF, in order to support the sharing of key health information and enable optimal care planning and delivery.

\subsubsection{Addressing Educational Needs}

Participants acknowledged the diversity in learning needs of professional, regulated and unregulated staff that need to be considered when designing and delivering HF education in LTC. Needs were identified for basic knowledge about HF and its management, and how such knowledge could be used to inform and sustain clinical processes specific for HF management in LTC. Educational needs also included the management of LTC residents with HF and other complex comorbidities.

\subsubsection{Providing Additional Resources}

Resources that were recommended to improve HF care management in LTC included comprehensive resident profiles that accompany the resident upon entry into LTC from the community or acute care setting, improved documentation and reporting systems with accurate resident information, and interprofessional involvement in which disciplines such as pharmacy, dietary, activation, social work and recreation collaborate in the care process. There was a recognized need for timely access to specific medical and diagnostic equipment such as x-ray devices and portable echocardiography, as well as improved access to lab services, in order to further improve HF 
management in the LTC setting.

\section{DISCUSSION}

HF is common in LTC and is associated with a significant burden of morbidity, health service utilization and mortality [2-6]. While the CCS HF guidelines offer recommendations on the care of complex older persons with HF, the optimal manner for implementing these guidelines in the Ontario LTC settings is unclear. This study identified several barriers to overcome in order to more effectively manage HF in LTC, including barriers related to inadequate HF knowledge among LTC staff, inadequate human and diagnostic resources, and poor communication both within LTC homes and between LTC and other sectors of the health care system. Staff working in LTC recommended improved communication strategies, better interprofessional relationships, addressing educational needs of LTC staff, and providing additional resources as organizational changes that will improve the management of HF.

The most fundamental barriers to overcome are related to interprofessional relationships and communication. These study findings suggest that an interprofessional collaborative approach to care could facilitate care processes related to HF management. It acknowledges that all those who provide care are active members of the care team and that better resident outcomes can be achieved by optimizing the expertise of all the team members involved in the care process [29]. Implementation of an interdisciplinary protocol to manage congestive $\mathrm{HF}$ in one American LTC home resulted in improved care based on certain quality indicators, including use of angiotensin converting enzyme inhibitors, nursing assessment, and symptom management [16]. Improved interprofessional collaboration has been identified as a key strategy to improve LTC resident care for many conditions, such as pain management and palliative care [34, 35]. By facilitating interprofessional collaborative practice, more efficient and effective use of health care providers' skills may be realized [36].

These findings also highlight the changing, more complex profile of LTC residents and the inherent challenges to managing multiple co-morbidities [26,27]. At the same time, the profile of LTC staff mix is changing, with fewer RNs in the staff mix and the majority of their time consumed by completing administrative tasks [37, 38]. These changes in LTC are reflected in our study results, where staff identified challenges with caring for complex residents but having little training and knowledge to do so. An appropriate mix of RNs, RPNs, and unregulated care providers is essential to ensure quality of care, particularly with respect to managing heart failure $[39,40]$.
The importance of PSWs cannot be overstated. PSWs spend more time at the bedside than any other care provider but receive the least amount of training [41]. However, they are often excluded from continuing education initiatives in their facilities or not supported by their employers to attend outside educational initiatives [41] Our study results add to the body of knowledge that PSWs are an integral part to the interdisciplinary team but who must be provided with the skills and training required to work effectively within a supportive environment.

There are some limitations to this study. As with all qualitative research studies, the Hawthorne effect and social desirability bias had the potential to influence participant responses during focus groups [42]. Researcher bias is a potential concern when interpreting qualitative data. However, the triangulation of methods, peer review, and member checking techniques designed in this study, as well as the separate analysis of each transcript by two researchers and collaboration for consensus on themes and results minimized the risk of bias. The small sample of homes may limit the generalizability of findings to other settings, however purposive sampling may have resulted in a fairly representative selection of homes and data saturation was achieved suggesting that important themes were captured.

\section{CONCLUSION}

Sustainable care processes for managing HF in LTC must reflect the capabilities and skill sets of all LTC staff, take into account the characteristics inherent to LTC residents and the LTC setting, and bring minimal disruption to existing work routines. In this paper, we report on the results of multiple focus groups among LTC staff roles, findings that will inform the development and implementation of HF care processes specific to Canadian LTC homes. The complexities of the LTC environment, including staffing hierarchies and regulatory pressures, present challenges to the implementation of care processes required to improve outcomes among LTC residents with HF. Capitalizing on a universal desire to provide HF treatment within LTC, leveraging existing successful practices, supporting resident autonomy, providing meaningful education that is tailored to each LTC staff role, and, most importantly, addressing the fundamental importance of successful interprofessional communication must be taken into consideration in order to optimally manage HF in LTC.

\section{ACKNOWLEDGEMENTS}

This project was supported by an unrestricted grant-in-aid from the Heart and Stroke Foundation of Ontario. The authors wish to acknowledge the other co-investigators who contributed to the design of this 
study, including Dr. Erin Tjam, Ms. Sonja Habjan, and Ms. Mary-Lou van der Horst. We wish to acknowledge the administrative support from Ms. Nisreen Murad, Ms. Christie Gennings, Ms. Linda Nash, and Mrs. Jessica Chiovitte Koski.

\section{REFERENCES}

[1] Sharkey, S. (2008) People caring for people: Impacting the quality of life and care of residents of long-term care homes. A report of the independent review of staffing and care standards for long-term care homes in Ontario. Saint Elizabeth Health Care.

[2] Daamen, M.A., Schols, J.M., Jaarsma, T. and Hamers, J.P. (2010) Prevalence of heart failure in nursing homes: A systematic literature review. Scandinavian Journal of Caring Sciences, 24, 202-208. doi:10.1111/j.1471-6712.2009.00708.x

[3] Heckman, G.A., Demers, C., McKelvie, R.S. and Hogan, D.B. (2007) Heart failure in older persons. Canadian Journal of General Internal Medicine, 2, 24-26.

[4] Wang, R., Mouliswar, M., Denman, S. and Kleban, M. (1998) Mortality of the institutionalized old-old hospitalized with congestive heart failure. Archives of Internal Medicine, 158, 2464-2468.

doi:10.1001/archinte.158.22.2464

[5] Heckman, G.A., Misiaszek, B., Merali, F., Turpie, I.D., Patterson, C.J., Flett, N. and McKelvie, R.S. (2004) The management of heart failure in Canadian long-term care facilities. Canadian Journal of Cardiology, 20, 963-969.

[6] Canadian Institute for Health Information (2009) Patient pathways: Transfers from continuing care to acute care. http://secure.cihi.ca/cihiweb/products/Patient_Transfers_ EN.pdf

[7] Inouye, S.K. (2006) Delirium in older persons. The New England Journal of Medicine, 354, 1157-1165. doi:10.1056/NEJMra052321

[8] Finucane, P., Wundke, R., Whitehead, C., Williamson, L. and Baggoley, C. (2000) Use of in-patient hospital beds by people living in residential care. Gerontology, 46, 133138. doi: $10.1159 / 000022148$

[9] Bowman, C.E., Elford, J., Dovey, J., Campbell, S. and Barrowclough, H. (2001) Acute hospital admissions from nursing homes: Some may be avoidable. Postgraduate Medical Journal, 77, 40-42. doi:10.1136/pmj.77.903.40

[10] Coburn, A.F., Keith, R.G. and Bolda, E.J. (2002) The impact of rural residence on multiple hospitalizations in nursing facility residents. Gerontologist, 42, 661-666. doi:10.1093/geront/42.5.661

[11] Finn, J.C., Flicker, L., Mackenzie, E., Jacobs, I.G., Fatovich, D.M., Drummond, S., Harris, M., Holman, D.C. and Sprivulis, P. (2006) Interface between residential aged care facilities and a teaching hospital emergency department in western Australia. Medical Journal of Australia, 184, 432-435.

[12] Rizza, P., Bianco, A., Pavia, M. and Angelillo, I.F. (2007) Preventable hospitalization and access to primary health care in an area of southern Italy. BMC Health Services
Research, 7, 134. doi:10.1186/1472-6963-7-134

[13] Colón-Emeric, C.S., Lekan, D., Utley-Smith, Q., Ammarell, N., Bailey, D., Corazzini, K., Piven, M.L. and Anderson, R.A. (2007) Barriers to and facilitators of clinical practice guideline use in nursing homes. Journal of the American Geriatrics Society, 55, 1404-1409. doi:10.1111/j.1532-5415.2007.01297.x

[14] Valle, R., Chinellato, M., Milani, L. and di Piave, S.D. (2001) Impact of a guideline-based management on outcomes of very old persons with heart failure living in nursing homes. Archives of Internal Medicine, 161, 22642265. doi:10.1001/archinte.161.18.2264-a

[15] American Medical Directors Association (2002) Heart failure: Clinical practice guideline.

[16] Martinen, M. and Freundl, M. (2004) Managing congestive heart failure in long-term care. Journal of Gerontological Nursing, 30, 5-12.

[17] Jacobs, B. (2011) Reducing heart failure hospital readmissions from skilled nursing facilities. Professional Case Management, 16, 18-24.

[18] Boxer, R.S., Dolansky, M.A., Frantz, M.A., Prosser, R., Hitch, J.A. and Piña, I.L. (2012) The Bridge Project: Improving heart failure care in skilled nursing facilities. Journal of the American Medical Directors Association, 13, e1-e7.

[19] Tjam, E.Y., Pletz, D.E., Hirdes, J.P., Smith, S.J., Costigan, J., Wilwerth, B. and Poss, J. (2006) Prevention of avoidable acute care use by retirement and long term care residents: Management of chronic heart failure through the use of an innovative inter-disciplinary education program for care providers (EPOCH study). Presented at the Canadian Cardiovascular Society, Vancouver.

[20] Newhouse, I.J., Heckman, G., Harrison, D., D’Elia, T., Kaasalainen, S., Strachan, P.H. and Demers, C. (2012) Barriers to the management of heart failure in Ontario long-term care homes: An interprofessional care perspective. Journal of Research in Interprofessional Practice and Education, in Press.

[21] Arnold, J.M.O., Liu, P., Demers, C., Dorian, P., Giannetti, N., Haddad, H., Heckman, G.A., Howlett, J.G., Ignaszewski, A., Johnstone, D.E., Jong, P., McKelvie, R.S., Moe, G.W., Parker, J.D., Rao, V., Ross, H.J., Sequeria, E.J., Svendsen, A.M., Teo, K., Tsuyuki, R.T. and White, M. (2006) Canadian cardiovascular society consensus conference recommendations on heart failure 2006: Diagnosis and management. Canadian Journal of Cardiology, 22, 23-45. doi:10.1016/S0828-282X(06)70237-9

[22] Morgan, D. (1997) Focus groups as qualitative research. 2nd Edition, Sage Publications, London.

[23] Sandelowski, M. (2000) Whatever happened to qualitative description? Research in Nursing \& Health, 23, 334340.

doi:10.1002/1098-240X(200008)23:4<334::AID-NUR9> 3.0.CO;2-G

[24] DiCenso, A., Hutchison, B., Grimshaw, J., Edwards, N. and Guyatt, G. (2005) Health services interventions. In: DiCenso, A., Guyatt, G. and Ciliska, D., Eds., Evidencebased nursing: A guide to practice, Elsevier Mosby, St. 
Louis.

[25] Coburn, A.F., Keith, R.G. and Bolda, E.J. (2002) The impact of rural residence on multiple hospitalizations in nursing facility residents. The Gerontologist, 42, 661-666. doi:10.1093/geront/42.5.661

[26] Hutt, E., Frederickson, E., Ecord, M. and Kramer, A.M. (2003) Associations among processes and outcomes of care for medicare nursing home residents with acute heart failure. Journal of the American Medical Directors Association, 4, 195-199. doi:10.1016/S1525-8610(04)70345-X

[27] McGrail, K.M., McGregor, M.J., Cohen, M., Tate, R.B. and Ronald, L.A. (2007) For-profit versus not-for-profit delivery of long-term care. Canadian Medical Association Journal, 176, 57-58. doi:10.1503/cmaj.060591

[28] Jogerst, G.J., Daly, J.M., Dawson, J.D., Peek-Asa, C. and Schmuch, G. (2006) Iowa nursing home characteristics associ- ated with reported abuse. Journal of the American Medical Directors Association, 7, 203-207. doi:10.1016/j.jamda.2005.12.006

[29] Weech-Maldonado, R., Meret-Hanke, L., Neff, M.C. and Mor, V. (2004) Nurse staffing patterns and quality of care in nursing homes. Health Care Management Review, 29, 107-116. doi:10.1097/00004010-200404000-00004

[30] Huberman, A.M. and Miles, M.B. (1994) Data management and analysis methods. In: Miles, M.B. and Huberman, A.M., Eds., Qualitative data analysis: An expanded sourcebook, 2nd Edition, Sage, Thousand Oaks, 428-444.

[31] Charmaz, K. (2006) Constructing grounded theory. Sage Publications, Thousand Oaks.

[32] Creswell, J. (2009) Research design: Qualitative, quantitative and mixed method approaches. 3rd Edition, Sage Publications, Thousand Oaks.

[33] Peshkin, A. (2000) The nature of interpretation in qualitative research. Educational Researcher, 29, 5-9. doi:10.3102/0013189X029009005

[34] Kaasalainen, S., Brazil, K., Coker, E., Ploeg, J., Martin-
Misener, R., Donald, F., DiCenso, A., Hadjistavropoulos, T., Dolovich, L., Papaioannou, A., Emili, A. and Burns, T. (2010) An action-based approach to improving pain management in long-term care. Canadian Journal on Aging, 29, 503-517. doi:10.1017/S0714980810000528

[35] Kortes-Miller, K., Habjan, S., Kelley, M.L. and Fortier, M. (2007) Development of a palliative care education program in rural long-term care facilities. Journal of palliative care, 23, 154-162.

[36] Ontario Ministry of Health and Long-Term Care (2005) Laying the foundation for change: A progress report on Ontario's health human resources initiatives. http://www.health.gov.on.ca/english/public/pub/ministry_ reports/hhr_05/hhr_05.html

[37] Canadian Nurses Association (2010) Patient safety: Developing the right staff mix. http://www.cna-aiic.ca/CNA/documents/pdf/publications/ PatientSafety_ThinkTank_e.pdf

[38] Canadian Nurses Association (2010) Evaluation framework to determine the impact of nursing staff mix decisions.

[39] Kaasalainen, S., Agarwal, G., Dolovich, L., Papaioannou, A., Brazil, K. and Akhtar-Danesh, N. (2010) Nurses' perception of their medication management system in longterm care. Canadian Journal of Nursing Research, 42, 58-79.

[40] McGillis-Hall, L. (2003) Nursing staff mix models and outcomes. Journal of Advanced Nursing, 44, 217-226. doi:10.1046/j.1365-2648.2003.02786.x

[41] Stolee, P., Esbaugh, J., Aylward, S., Cathers, T., Harvey, D.P., Hillier, L.M., Keat, N. and Feightner, J.W. (2005) Factors associated with the effectiveness of continuing education in long-term care. Gerontologist, 45, 399-405. doi:10.1093/geront/45.3.399

[42] Parsons, H.M. (1974) What happened at Hawthorne? Science, 183, 922-932. doi:10.1126/science.183.4128.922 